Journal of Engineering and Applied Sciences 15 (1): 303-309, 2020

ISSN: 1816-949X

(C) Medwell Journals, 2020

\title{
Experimental Model of Distributed Service Broker Policy Algorithm in Cloud based Centralized and Distributed Data Center
}

\author{
${ }^{1}$ Shivani Dubey, ${ }^{2}$ Mamta Dahiya and ${ }^{3}$ Sunayana Jain \\ ${ }^{1}$ JSS Academy of Technical Education, Department of Computer Applications, Noida, India \\ ${ }^{2}$ School of Engineering and Technology, Ansal University, Gurugaon, India \\ ${ }^{3}$ Department of Operation and Information Technology, \\ Institute of Management Studies, Ghaziabad, India
}

\begin{abstract}
In recent years cloud computing is a very advanced technique to distribute workload among all the data centers and also balance those data center very smoothly. To improve the performance of data centers, load balancing is used to distribute workload of arrival requests on data centers equally in the computing environment. Load balancing has aim to minimize the response time among the users from different data centers and also improve resource utilization by using cloud resources. Cloud based data centers always require efficient load balancing strategies to reduce work load on Virtual Machines (VM). Researchers proposed various load balancing algorithms to optimize different parameters. In this study, we are proposing a distributed service broker policy algorithm which gives better result in centralized and distributed data center environment, considers cloud resources for specific demands and also reduces work load on virtual machines. There are so many VM load balancing algorithms have compared by using different data center broker policies. A cloud analyst simulator, simulate these algorithms and then accurate result will be showed. Experimental results have shown that our proposed Distributed Service Broker Policy (DSBP) algorithm in terms of execution time, response time, throughput, cost as compared to existing round robin, active monitoring and throttled algorithms. The experiments have been done using cloud analyst simulator and comparative analysis is evaluated based on our proposed DSBP algorithm and the results are presented in detail.
\end{abstract}

Key words: Load balancing algorithms, DSBP, cloud analyst, workload, strategies, parameters

\section{INTRODUCTION}

Cloud computing provides an easiest way for saving and retrieving data when people want to create a large database set with a large file size for huge number of users at different locations in the world (Brar et al., 2014). The enormous requests of different located users give the impact on the server with overloading. So, we require the accurate solution for overloading problem on different data center for distributing load across multiple data centers. Distributed computing has various advantages for user's requirements and applications (Dave and Maheta, 2014). In centralized data center all load is allocated and scheduled on single data center so this data center is used for sharing cloud network and can apply static and dynamic load balancing approaches. These approaches reduce the response time required to analyze different cloud resources but it generates higher overloading on the centralized data center and this time recovery will be not easy in case of failure of data center. In distributed data center, there is no even one data center is responsible for managing scheduling decision and balancing load on computer network instead distributed data centers manage cloud network to generate accurate load balancing approach. In distributed data center environment, failure of data center is not overloaded to manage load balancing decision. The distributed algorithm removes the obstacles by the centralized data center approaches and improves reliability, stability and efficiency of cloud network. Load balancing is imperative for distributed environments. Cloud based load balancing provides finest services for user's requests to ensure that no single data center is overloaded. It helps in proper utilization of resources. It also transfers the extra load to other data centers (Patel and Chauhan, 2015). Load balancing is required to implement the performance of cloud based data centers by minimize the completion time, data transfer cost and ignore the heavy load on the data centers (Dsouza et al., 2016). The implementation of proposed algorithm has been done using cloud analyst and result is based on comparative analysis to evaluate the better result between the centralized and distributed data center. 
J. Eng. Applied Sci., 15 (1): 303-309, 2020

Table 1: Existing results of various load balancing algorithms

\begin{tabular}{|c|c|c|c|}
\hline Researchers & Algorithm description & Response time & Data transfer cost \\
\hline Randles et al. (2010) & $\begin{array}{l}\text { Proposed load balancing approaches by Honeybee for aging } \\
\text { behaviour based random sampling and active clustering }\end{array}$ & No & Yes \\
\hline Gulati and Chopra (2013) & $\begin{array}{l}\text { Presented a comparative survy of load balancing algorithms with } \\
\text { respect to scalability and process migration }\end{array}$ & Yes & No \\
\hline Sidana et al. (2016) & $\begin{array}{l}\text { Presented NBST algorithm for load balancing by sort speed of } \\
\text { VMs and sort the length of the cloud }\end{array}$ & No & No \\
\hline Tang et al., (2016) & $\begin{array}{l}\text { Proposed DLBS load balance scheduling algorihm to balance } \\
\text { stream through time slots }\end{array}$ & No & No \\
\hline Mishra et al. (2017) & $\begin{array}{l}\text { Defined anovel load balancing approach to virtualized resources } \\
\text { of the data center efficiently }\end{array}$ & Yes & No \\
\hline $\begin{array}{l}\text { Omranian-Khorasani and } \\
\text { Naghibzadeh (2017) }\end{array}$ & $\begin{array}{l}\text { Presented DCLB algorithm to refine deadline distribution for } \\
\text { lower communication cost }\end{array}$ & No & Yes \\
\hline Dhari and Arif (2017) & $\begin{array}{l}\text { Proposed te LDAB scheduling algorithm to implement load } \\
\text { balancing to perform a task on each virtual machine }\end{array}$ & No & No \\
\hline Putra et al. (2017) & $\begin{array}{l}\text { Defined load balancing approach reduces the response time } \\
\text { and latency to copy data from the source VM to the target }\end{array}$ & Yes & No \\
\hline
\end{tabular}

Background and challenges: Cloud computing is a new generation model to manipulate, construct and access for distributing different applications over the internet. In cloud computing, multiple systems are used to fulfill the user's requirements by using different resources. Cloud computing is used for dispatching the targets to resources which are considered by the multiple systems. There are so many challenges in research of cloud computing such as access control at different locations, security, data on time and major challenge is load balancing. Cloud computing has added many features for balancing loads among the data centers. Load balancing calculate the workload, minimize execution time, response time, latency, time delay and maximize utilization of resources and throughput (Tripathi and Singh, 2017). Wang et al. (2011) proposed a two level architecture based on VM algorithm which is evaluated for applications and VM migration for selecting best VM among the data centers (Wang et al., 2011). Tian et al. (2011) proposed a DAIRS load balancing and scheduling algorithm which is used for balancing the workload in cloud based data center according to the three parameters; CPU, memory and network bandwidth (Tian et al., 2011). Ma et al. (2012) defined a model of distributed load balancing allocation of VM cloud based data center by using the TOPSIS method which considered multi criteria decision making techniques in which data center runs a module of VM that investigates the local resource utilization (Chen and Zhang, 2012). Chen and Zhang (2012) proposed set based centralized method which handles workflow scheduling issue in cloud and which is also enable for QoS parameters: Deadline, budget, availability and reliability (Simjanoska et al., 2013). Simjanoska et al. (2013) proposed a L3B centralized Archbased load balancer solution for load balancing which works between the users and cloud heterogeneous data centers (Ma et al., 2012). Xu et al. (2013) also presented an Archbased which controls the communication with the divided balancers and each division uses a local balancer that selects the best approach for load balancing. Khalili and Babamir (2015) mapped the scheduling the targets to run on existing resources that consider to increase utilization and make span. An optimize scheduling uses centralized algorithm to minimize the weights among the data centers.

In Table 1, so many load balancing algorithms are presented by the authors to determine the result of those algorithms on the basis of average response time and total cost of data center in cloud computing environment. We can see all results as per response time and cost are not reduced at same time otherwise not be counted. In our proposed DSBP algorithm, both are reducing and has minimum value of results in both centralized and distributed data center environment over the cloud.

\section{MATERIALS AND METHODS}

Existing algorithms: Load balancing is a technique to distribute the excess workload across all the data centers. Load balancing is used to achieve a better service provisioning and resource utilization ratio for improving the overall performance of the system. Incoming requests coming from different location are received by the load balancer for distributing to the different data centers for accurate load distribution (Kashyap and Viradiya, 2014). An overview of related works on various scheduling and load balancing algorithms, the major problems being investigated on proposed distributed broker policy algorithm is its ability to achieve better response time and data transfer cost. Therefore, this study considers the improvement of round robin algorithm, active monitoring algorithm and throttled algorithm and proposed our algorithm which leads to better resource utilization. 
- Round robin algorithm: Round Robin is a load balancing algorithm which divides processes among all the data centers. The process allocation system is managed locally independent from the remote data centers. Round robin algorithm has fixed quantum time which creates time limitation. In round robin algorithm, the load balancer allocates a VM for requesting data center in cyclic way among all connected data centers (Supreeth and Biradar, 2013).

- Active monitoring algorithm: Active monitoring maintains the requests on each VM and the number of request currently allocated to which $\mathrm{VM}$, when a request is allocate to new $\mathrm{VM}$, active monitoring returns the VM id to data centers controller. The data center controller sends the request to the VM identified by VM id. The data center controller specifies to active monitoring to new allocated request and cloudlets is sent to it (Nema and Edwin, 2016)

- Throttled load balancing algorithm: Throttled load balancing algorithm calculation is based on appropriate VMs for controlling a specific occupation. The job manager has rundown of every single VM, utilizes the order and allocates the specified work given by the user. According to request of user, if job is fine for specific VM on the data center, then job is assigned to appropriate VM. If there is no VM accessible to send acknowledgement to the jobs, the job manager queued the requests (Asha et al., 2018)

\section{Proposed DSBP algorithm}

DSBP algorithm: The DSBP is implementation of throttled load balancing algorithm. The DSBP accommodates the existing requirements by analyzing real time scenario to evaluate the processing time to minimize the response time by the policy broker. In this algorithm, job processing time is depended on the computational task to be represented. DSBP evaluates the size of job as per required processing time and works as expected even though under the capacity of each VMs are different because each VM has different hardware configuration.

DSBP load balancer algorithm:

1. DSBP load balancer performs load balancing by update, maintain index tables:

(Available Index $=0$ \& Busy Index $=1$ )

2. The Data Center Controller receives a new request

3. Data Center Controller queries to DSBP load balancer for nearest next allocations

4. DSBP load balancer detects and sends VM Id (VM) across data center for available Index table of nearest Data Center Controller

- Data Center Controller sends the request with VM Id

- Data Center Controller informs the DSBP load Balancer for a new allocation

- $\quad$ DSBP load Balancer will update VM into the busy Index and wait for the new request from Data Center Controller

5. If the table " Available Index " is empty then DSBP load balancer will return a value NULL to the Data Center Controller and Data Center Controller arranges the request

6. After executing the request, Data Controller gets response from $\mathrm{VM}$, it will notify to the load balancer then update the " Available Index" table

7. If there are multiple requests the Data Center Controller repeats STEP 3 and the process is again repeated

\section{RESULTS AND DISCUSSION}

Cloud analyst defines the world into the six region that correspond with six main lands. The simulation setup and result analysis are evaluated for $60 \mathrm{~min}$ by multiple number of users in centralized and distributed center environment which have separately VMs (Fig. 1).

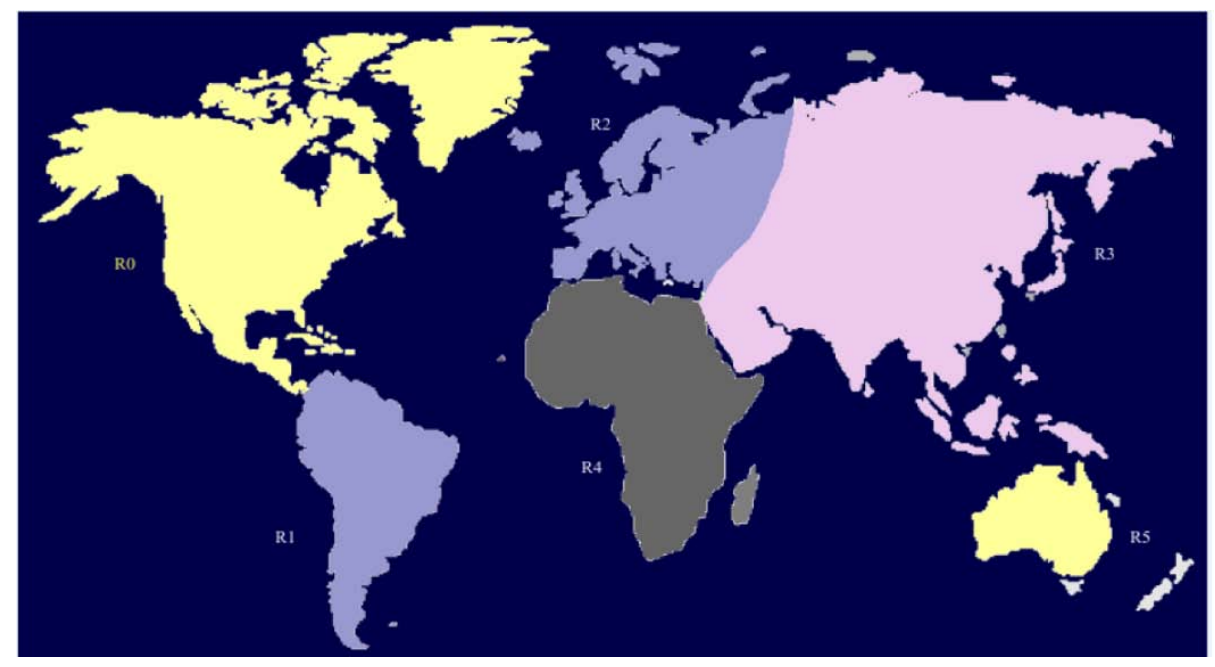

Fig. 1: Main screen display on cloud analyst 

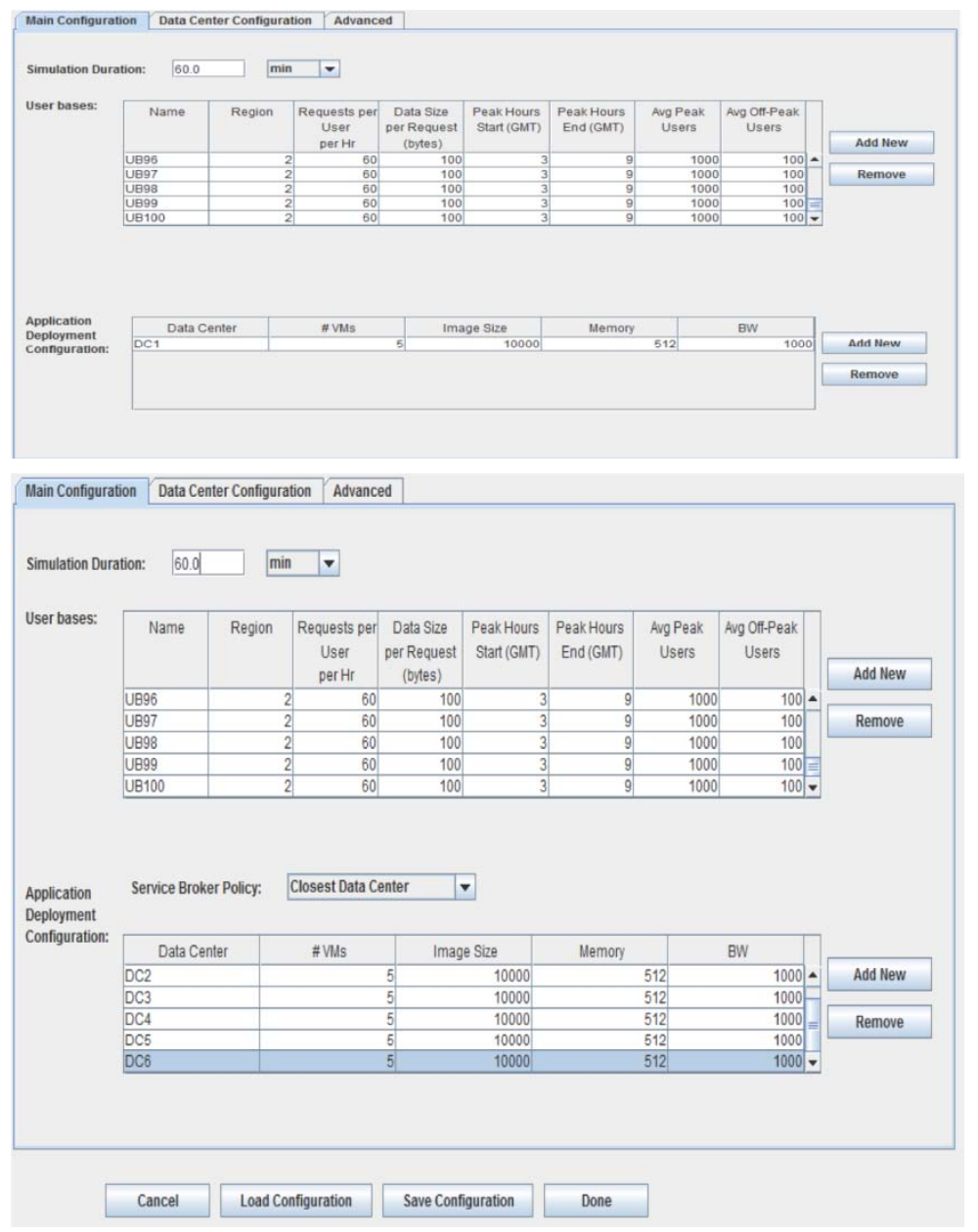

\section{Delay Matrix}

The transmission delay between regions. Units in milliseconds

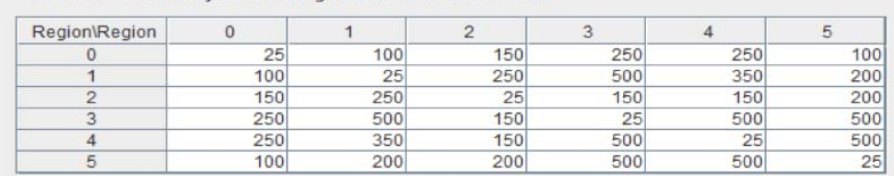

Bandwidth Matrix

The available bandwidth between regions for the simulated application. Units in Mbps

\begin{tabular}{|c|r|r|r|r|r|r|}
\hline RegionlRegion & \multicolumn{1}{|c|}{0} & \multicolumn{1}{|c|}{1} & \multicolumn{1}{|c|}{2} & \multicolumn{1}{|c|}{3} & \multicolumn{1}{c|}{4} & \multicolumn{1}{c|}{5} \\
\hline 0 & 2,000 & 1,000 & 1,000 & 1,000 & 1,000 & 1,000 \\
\hline 1 & 1,000 & 800 & 1,000 & 1,000 & 1,000 & 1,000 \\
\hline 2 & 1,000 & 1,000 & 2,500 & 1,000 & 1,000 & 1,000 \\
\hline 3 & 1,000 & 1,000 & 1,000 & 1,500 & 1,000 & 1,000 \\
\hline 4 & 1,000 & 1,000 & 1,000 & 1,000 & 500 & 1,000 \\
\hline 5 & 1,000 & 1,000 & 1,000 & 1,000 & 1,000 & 2,000 \\
\hline
\end{tabular}

\begin{tabular}{|l|}
\hline Done \\
\hline
\end{tabular}

Fig. 2: Cloud analyst simulator

The classes of cloud analyst simulator have been extended to utilize newly proposed DSBP algorithm. In Fig. 2, the response time and load are analyzed by using methods of round robin, active monitoring, throttled and DSBP algorithm for distributed data center. 


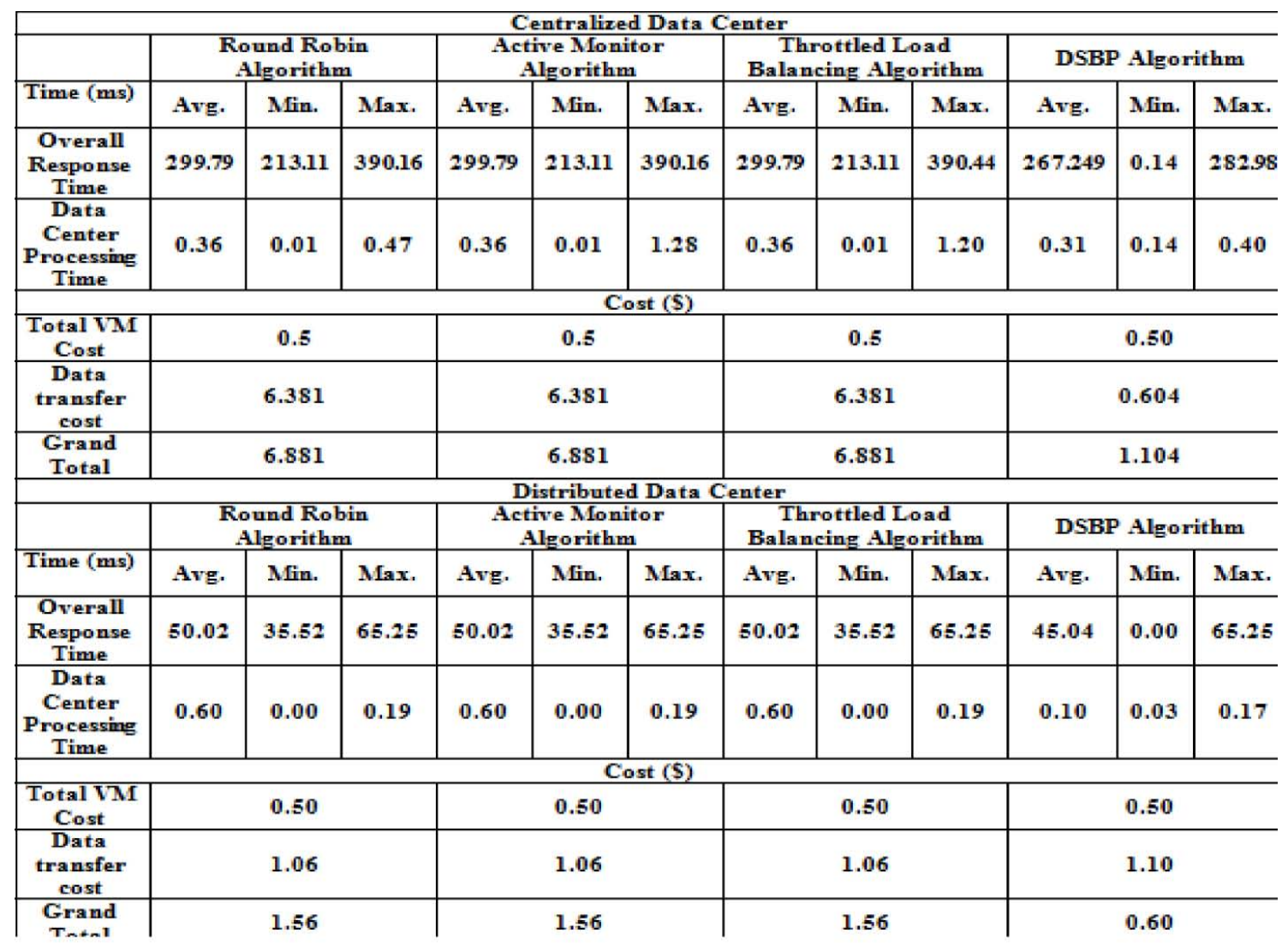

Fig. 3: Values of all existing and proposed DSBP algorithm

This will start the simulation and the progress bar at the top of the simulation panel shows the percentage completion of the simulation. The simulation screen will display a simple animation showing which user bases are sending messages to which data centers. A simulation can be cancelled before the completion of the run using the cancel button at the bottom right hand corner. It may take a while after clicking the cancel button for the simulation to halt as it will continue to gather the simulation data of the requests that had been generated before cancelling but had not been completed.

After performance different experiments by cloud analyst successfully, we get overall response time of different load balancing algorithms as given in Fig. 3. Analytical result of overall response time and load based on various algorithms in loud computing environment is shown below:

Figure 3 represents the values of all existing algorithms and proposed DSBP algorithm for overall response time from different data centers which shows DSBP has better performance showing in graph.

\section{CONCLUSION}

Load balancing attains maximum use of resources. As far as our proposed DSBP algorithm provides best results when working data centers are centralized and distributed environment. In this study we discussed various load balancing.

In this study, we discussed various load balancing schemes, each having some pros and cons. On one hand static load balancing scheme provide easiest simulation and monitoring of environment but fail to model heterogeneous nature of cloud. On the other hand, dynamic load balancing algorithm are difficult to simulate but are best suited in heterogeneous environment of cloud computing. Also, the level at node which implements this static and dynamic algorithm plays a vital role in deciding the effectiveness of algorithm. Unlike centralized algorithm, distributed nature of algorithm provides better fault tolerance but requires higher degree of replication and on the other hand, hierarchical algorithm divide the load at different levels of hierarchy with upper level nodes requesting for services of lower level nodes in balanced manner. Hence, dynamic load balancing techniques in 
distributed or hierarchical environment provide better performance. However, performance of the cloud computing environment can be further maximized if dependencies between tasks are modeled using workflows.

\section{REFERENCES}

Asha, V., B. Kumar and V. Girish, 2018. Load balancing in cloud computing. Int. J. Recent Trends Eng. Res., 4: 118-125.

Brar, H.S., V. Thapar and K. Kishor, 2014. A survey of load balancing algorithms in cloud computing. Int. J. Comput. Sci. Trends Technol., 2: 103-106.

Chen, W.N. and J. Zhang, 2012. A set-based discrete PSO for cloud workflow scheduling with user-defined QoS constraints. Proceedings of the 2012 IEEE International Conference on Systems, Man and Cybernetics (SMC), October 14-17, 2012, IEEE., Seoul, South Korea, pp: 773-778.

Dave, S. and P. Maheta, 2014. Utilizing round robin concept for load balancing algorithm at virtual machine level in cloud environment. Int. J. Comput. Applic., 94: 23-29.

Dhari, A. and K.I. Arif, 2017. An efficient load balancing scheme for cloud computing. Indian J. Sci. Technol., Vol. 10, No. 11. 10.17485/ijst/2017/v10i11/110107.

Dsouza, M., M. Rizwan, R. Gaonkar and S. Sathyanarayana, 2016. Scheduling and load balancing techniques in cloud computing: A survey. Int. J. Latest Trends Eng. Technol., 2016: 309-316.

Gulati, A. and R.K. Chopra, 2013. Dynamic round robin for load balancing in a cloud computing. Int. J. Comput. Sci. Mobile Comput., 2: 274-278.

Kashyap, D. and J. Viradiya, 2014. A survey of various load balancing algorithms in cloud computing. Int. J. Sci. Technol. Res., 3: 115-119.

Khalili, A. and S.M. Babamir, 2015. Makespan improvement of PSO-based dynamic scheduling in cloud environment. Proceedings of the 2015 23rd Iranian Conference on Electrical Engineering, May 10-14, 2015, IEEE., Tehran, Iran, pp: 613-618.

Ma, F., F. Liu and Z. Liu, 2012. Distributed load balancing allocation of virtual machine in cloud data center. Proceedings of the 2012 IEEE International Conference on Computer Science and Automation Engineering, June 22-24, 2012, IEEE., Beijing, China, pp: 20-23.
Mishra, S.K., M.A. Khan, B. Sahoo, P. Deepak, M.S. Obaidat and K.F. Hsiao, 2017. Time efficient dynamic threshold-based load balancing technique for cloud computing. Proceedings of the International Conference on Computer, Information and Telecommunication Systems, CITS, July 21-23, 2017, IEEE., Dalian, China, pp: 161-165.

Nema, R. and S.T. Edwin, 2016. A new efficient virtual machine load balancing algorithm for a cloud computing environment. Int. J. Latest Res. Eng. Technol., 2: 69-75.

Omranian-Khorasani, S. and M. Naghibzadeh, 2017. Deadline constrained load balancing level based workflow scheduling for cost optimization. Proceedings of the 2nd IEEE International Conference on Computational Intelligence and Applications, (ICCIA), September 8-11, 2017, IEEE., Beijing, China, pp: 113-118.

Patel, N. and S. Chauhan, 2015. A survey on load balancing and scheduling in cloud computing. Int. J. Innovative Res. Sci. Technol., 1: 185-189.

Putra, J.P., S.M.S. Nugroho and I. Pratomo, 2017. Live migration based on cloud computing to increase load balancing. Proceedings of the International Seminar on Intelligent Technology and Its Application, (ISITIA), August 28-29, 2017, IEEE., Surabaya, Indonesia, pp: 286-290.

Randles, M., D. Lamb and A. Taleb-Bendiab, 2010. A comparative study into distributed load balancing algorithms for cloud computing. Proceedings of the 24th IEEE International Conference on Advanced Information Networking and Applications Workshops, April 20-23, 2010, IEEE., Perth, WA, Australia, pp: 551-556.

Sidana, S., N. Tiwari, A. Gupta and I.S. Kushwaha, 2016. NBST algorithm: A load balancing algorithm in cloud computing. Proceedings of the International Conference on Computing, Communication and Automation (ICCCA), April 29-30, 2016, IEEE., Noida, India.

Simjanoska, M., S. Ristov, G. Velkoski and M. Gusev, 2013. L3B: Low level load balancer in the cloud. Proceedings of the International Conference on Eurocon, July 1-4, 2013, IEEE., Zagreb, Croatia, pp: 250-257.

Supreeth, S. and S. Biradar, 2013. Scheduling virtual machines for load balancing in cloud computing platform. Int. J. Sci. Res., 2: 437-441.

Tang, F., L.T. Yang, C. Tang, J. Li and M. Guo, 2016. A dynamical and load-balanced flow scheduling approach for big data centers in clouds. IEEE Trans. Cloud Comput., 6: 915-928. 
Tian, W., Y. Zhao, Y. Zhong, M. Xu and C. Jing, 2011. A dynamic and integrated load-balancing scheduling algorithm for cloud datacenters. Proceedings of the 2011 IEEE International Conference on Cloud Computing and Intelligence Systems, September 15-17, 2011, IEEE., Beijing, China, pp: 311-315.

Tripathi, A.M. and S. Singh, 2017. A literature review on algorithms for the load balancing in cloud computing environments and their future trends. Comput. Model. N. Technol., 21: 64-73.
Wang, R., W. Le and X. Zhang, 2011. Design and implementation of an efficient load-balancing method for virtual machine cluster based on cloud service. Proceedings of the 4th IET International Conference on Wireless, Mobile \& Multimedia Networks, November 27-30, 2011, IET., Beijing, China, pp: 321-324.

Xu, G., J. Pang and X. Fu, 2013. A load balancing model based on cloud partitioning for the public cloud. Tsinghua Sci. Technol., 18: 34-39. 